TRANSACTIONS OF THE

AMERICAN MATHEMATICAL SOCIETY

Volume 356, Number 3, Pages 881-893

S 0002-9947(03)03452-4

Article electronically published on October 9, 2003

\title{
HILBERT SPACES OF DIRICHLET SERIES AND THEIR MULTIPLIERS
}

\author{
JOHN E. M ${ }^{\mathrm{c}} \mathrm{CARTHY}$
}

\begin{abstract}
We consider various Hilbert spaces of Dirichlet series whose norms are given by weighted $\ell^{2}$ norms of the Dirichlet coefficients. We describe the multiplier algebras of these spaces. The functions in the multiplier algebra may or may not extend to be analytic on a larger half-plane than the functions in the Hilbert space.
\end{abstract}

\section{INTRODUCTION}

Let $\mathbf{w}=\left\{w_{n}\right\}_{n=n_{0}}^{\infty}$ be a sequence of positive numbers. In this paper we are concerned with Hilbert spaces of functions representable by Dirichlet series

$$
\mathcal{H}_{\mathbf{w}}=\left\{f(s)=\left.\sum_{n=n_{0}}^{\infty} a_{n} n^{-s}\left|\|f\|_{\mathcal{H}_{\mathbf{w}}}^{2}:=\sum_{n=n_{0}}^{\infty}\right| a_{n}\right|^{2} w_{n}<\infty\right\} .
$$

The prototypical case, where $w_{n} \equiv 1, n \geq 1$, was first studied in a beautiful paper by H. Hedenmalm, P. Lindqvist and K. Seip [9]. Among other results, they characterized the multipliers of the space (see Theorem 1.11 below for a statement of their result).

One purpose of this paper is to consider the scale of spaces obtained from the weight sequences $\mathbf{w}^{\alpha}$, defined for $n \geq 2$ by

$$
w_{n}^{\alpha}=(\log n)^{\alpha} .
$$

For brevity, we shall write $\mathcal{H}_{\alpha}$ for the space $\mathcal{H}_{\mathbf{w}^{\alpha}}$; specifically

$$
\mathcal{H}_{\alpha}=\left\{f(s)=\left.\sum_{n=2}^{\infty} a_{n} n^{-s}\left|\sum_{n=2}^{\infty}\right| a_{n}\right|^{2}(\log n)^{\alpha}<\infty\right\} .
$$

(When $\alpha=0$, it is more natural to let $n_{0}=1$ and to include the constant functions in $\mathcal{H}_{0}$. It is not essential to any of the issues we discuss here.)

Before going further, let us remind the reader of some basic facts about Dirichlet series. A nice treatment can be found in Titchmarsh's book [16]. We shall follow the convention of writing the complex variable $s=\sigma+i t$. A Dirichlet series is a series of the form

$$
\sum_{n=1}^{\infty} a_{n} n^{-s}
$$

Received by the editors December 7, 2001.

2000 Mathematics Subject Classification. Primary 30B50, 46E20.

The author was partially supported by National Science Foundation Grant DMS 0070639. 
Such a series may converge for no values of $s$; if it converges for any particular $s_{0}$, then it converges for all $s$ with $\sigma>\Re\left(s_{0}\right)$. Therefore the largest open set in which a series ([0.3) converges is a half-plane (at what points on the boundary of the half-plane the series converges is, in general, a delicate question). Let us adopt the notation, for $\rho$ a real number, that $\Omega_{\rho}$ is the half-plane

$$
\Omega_{\rho}=\{s \in \mathbb{C} \mid \sigma>\rho\} .
$$

Let

$$
\sigma_{c}=\inf \left\{\Re(s): \sum_{n=1}^{\infty} a_{n} n^{-s} \text { converges }\right\}
$$

this is called the abscissa of convergence of the series. The largest domain of convergence of the series is $\Omega_{\sigma_{c}}$. There are three other abscissae associated with the series (0.3) which we shall need. The first is the abscissa of absolute convergence, $\sigma_{a}$, defined by

$$
\sigma_{a}=\inf \left\{\Re(s): \sum_{n=1}^{\infty} a_{n} n^{-s} \text { converges absolutely }\right\} .
$$

Obviously $\sigma_{a} \geq \sigma_{c}$; it is straightforward that $\sigma_{a} \leq \sigma_{c}+1$, because if $\sum_{n=1}^{\infty} a_{n} n^{-s}$ converges, then $\left|a_{n}\right| n^{-\sigma}=o(1)$. The second is the abscissa of boundedness $\sigma_{b}$, defined by

$$
\sigma_{b}=\inf \left\{\rho: \sum_{n=1}^{\infty} a_{n} n^{-s} \text { converges to a bounded function in } \Omega_{\rho}\right\} .
$$

The third abscissa is the abscissa of uniform convergence $\sigma_{u}$, defined by

$$
\sigma_{u}=\inf \left\{\rho: \sum_{n=1}^{\infty} a_{n} n^{-s} \text { converges uniformly in } \Omega_{\rho}\right\} .
$$

Clearly $\sigma_{u} \geq \sigma_{b}$; H. Bohr proved that $\sigma_{u}=\sigma_{b}$, and $\sigma_{a} \leq \sigma_{b}+\frac{1}{2}$ [7]. Note that the series does not necessarily define a bounded function in $\Omega_{\sigma_{b}}$, but the function it represents is bounded in all strictly smaller half-planes. If all the coefficients $a_{n}$ are positive, then all three of $\sigma_{c}, \sigma_{b}, \sigma_{a}$ coincide.

We shall let $\mathcal{D}$ denote the set of functions that can be represented in some halfplane by a Dirichlet series.

Let $f(s)$ be holomorphic in the half-plane $\Omega_{\rho}$. Let $\varepsilon>0$. A real number $\tau$ is called an $\varepsilon$ translation number of $f$ if

$$
\sup _{s \in \Omega_{\rho}}|f(s+i \tau)-f(s)| \leq \varepsilon .
$$

The function $f(s)$ is called uniformly almost periodic in the half-plane $\Omega_{\rho}$ if, for every $\varepsilon>0$, there exists a positive real number $M$ such that every interval in $\mathbb{R}$ of length $M$ contains at least one $\varepsilon$ translation number of $f$.

We shall need the following theorem. The proof can be found in [6, p. 144].

Theorem 0.4. Suppose that $f(s)$ is represented by a Dirichlet series that converges uniformly in the half-plane $\Omega_{\rho}$. Then $f$ is uniformly almost periodic in $\Omega_{\rho}$. 
Returning to the spaces $\mathcal{H}_{\alpha}$, it follows from the Cauchy-Schwarz inequality that any function in any space $\mathcal{H}_{\alpha}$ has $\sigma_{a} \leq \frac{1}{2}$. Moreover, for all $\varepsilon>0$, the function 1

$$
\zeta\left(\frac{1}{2}+\varepsilon+s\right)-1=\sum_{n=2}^{\infty} \frac{1}{n^{\frac{1}{2}+\varepsilon}} n^{-s}
$$

is in every $\mathcal{H}_{\alpha}$ and has a pole at $\frac{1}{2}-\varepsilon$, so the largest common domain of analyticity of the functions in any $\mathcal{H}_{\alpha}$ is $\Omega_{1 / 2}$. The reproducing kernel for $\mathcal{H}_{\mathrm{w}}$ is

$$
k(s, u)=\sum_{n=n_{0}}^{\infty} \frac{1}{w_{n}} n^{-s-\bar{u}} ;
$$

for the spaces $\mathcal{H}_{\alpha}$ this is essentially a fractional derivative or integral of the $\zeta$ function at $s+\bar{u}$.

We claim that the scale of spaces $\mathcal{H}_{\alpha}$ is in many ways analogous to the scale of spaces of holomorphic functions in the unit disk defined by

$$
\mathcal{K}_{\alpha}=\left\{g(z)=\left.\sum_{n=0}^{\infty} a_{n} z^{n}\left|\sum_{n=0}^{\infty}\right| a_{n}\right|^{2}(n+1)^{\alpha}<\infty\right\} .
$$

Thus the space $\mathcal{H}_{0}$ corresponds to the Hardy space, the space $\mathcal{H}_{-1}$ corresponds to the Bergman space, and the space $\mathcal{H}_{1}$ corresponds to the Dirichlet space in the setting of the disk.

Note first that differentiation is a unitary map from $\mathcal{H}_{\alpha}$ to $\mathcal{H}_{\alpha-2}$, and fractional differentiation/integration maps any $\mathcal{H}_{\alpha}$ to any $\mathcal{H}_{\beta}$. Although this is not exactly true for the spaces $\mathcal{K}_{\alpha}$ as we defined them, it is essentially true. It fails only because (a) differentiation annihilates the constant functions, and (b) the choice of norms in (0.6) does not quite render differentiation isometric. For example, the norm of $z^{n}$ in the Dirichlet space is $\sqrt{n+1}$, and the norm of $n z^{n-1}$ in the Bergman space is $\sqrt{n}$. Asymptotically these agree, and it is true as function spaces that the Dirichlet space is the set of functions whose derivatives are in the Bergman space.

The main reason for our analogy is the Plancherel formula (Theorem 1.5 below) which gives the norm of a function in $\mathcal{H}_{\alpha}$, for $\alpha>0$, in terms of a weighted area integral. As $\alpha$ tends to 0 , the measure tends to a line integral on the boundary.

Why study the $\mathcal{H}_{\alpha}$ spaces? We offer three considerations. First, a Plancherel formula calculating the norm of a function in two different ways frequently has serendipitous consequences (see e.g. 9], where they exploit it to answer an old question of A. Beurling). Second, the kernel functions for these spaces are formed from the zeta function and its relatives (0.5), and anything connected with the zeta function is, in the author's opinion, automatically interesting. Third, much of the interesting function theory on the disk is obtained by restricting functions to various spaces. These spaces are often naturally included in an $L^{p}$-scale in one direction (e.g. the Hardy scale $H^{p}$ ), and in another scale obtained by changing the measure $\left(e . g . \mathcal{K}_{\alpha}\right)$. The function theory of Dirichlet series is not nearly as advanced as the function theory of the unit disk, so the natural scale of Hilbert spaces seems to be a good starting point. In this paper we do not consider the $L^{p}$-scale, but changing the $L^{2}$-norm on the right-hand side of the Plancherel formula (1.6) gives the obvious candidate for the $H^{p}$-like norms. For some interesting results on $H^{p}$ spaces of Dirichlet series, we refer the reader to [5].

\footnotetext{
${ }^{1}$ We use $\zeta$ to denote the Riemann zeta-function: $\zeta(s)=\sum_{n=1}^{\infty} n^{-s}$.
} 
In Section 1 we study $\mathcal{H}_{\alpha}$ for $\alpha \leq 0$. These spaces are Bergman-like. The norm can be given as (a limit of) an $L^{2}$-norm of the function. We extend the theorem of Hedenmalm, Lindqvist and Seip [9] to show that for all these spaces the multiplier algebra is the same, viz. the space of all functions representable by Dirichlet series and bounded in $\Omega_{0}$ (Theorem 1.11).

In Section 2, we study $\mathcal{H}_{\alpha}$ for $\alpha>0$. These are Dirichlet-like spaces: functions whose derivatives of some fixed order are in a Bergman-like space. We give an incomplete characterization of the multiplier algebra (Corollary 2.4).

In Section 3, we consider a different choice of weight sequence. We let $w_{n}=\frac{1}{F(n)}$, where $F(n)$ is the number of ways $n$ can be factored with integer factors greater than 1 . The reproducing kernel is

$$
k(s, u)=\frac{1}{2-\zeta(s+\bar{u})} .
$$

This has the interesting property of being a complete Pick kernel, and several properties flow from this (see e.g. [2] for a discussion). We show that, unlike for the spaces $\mathcal{H}_{\alpha}$, the multipliers do not extend to be analytic on a larger domain than the domain of analyticity of the Hilbert space, by constructing an interpolating sequence for the multiplier algebra that accumulates on the boundary of the halfplan of convergence of the Hilbert space (Theorem 3.6]).

\section{BERGMAN-LIKE SPACES}

Throughout this section, $\mu$ will be a positive Radon measure on $[0, \infty)$ for which

$$
\int_{0}^{\infty} n_{0}^{-2 \sigma} d \mu(\sigma)=\int_{0}^{\infty} e^{-2\left(\log n_{0}\right) \sigma} d \mu(\sigma)<\infty
$$

for some positive integer $n_{0}$. We also assume that

$$
0 \text { is in the support of } \mu \text {. }
$$

We define $w_{n}$, for $n \geq n_{0}$, by

$$
w_{n}:=\int_{0}^{\infty} n^{-2 \sigma} d \mu(\sigma)
$$

Letting $\mu_{\alpha}$ be the measure

$$
d \mu_{\alpha}(\sigma)=\frac{2^{-\alpha}}{\Gamma(-\alpha)} \sigma^{-1-\alpha} d \sigma
$$

gives the weights from 0.2 for $\alpha<0$ :

$$
\int_{0}^{\infty} n^{-2 \sigma} d \mu_{\alpha}(\sigma)=(\log n)^{\alpha}, \quad n \geq 2, \alpha<0 .
$$

We let $\mu_{0}$ be the unit point mass at 0 , which has all of its moments equal to 1 .

For any measure satisfying (1.1) and (1.2), the moments $w_{n}$ are a decreasing sequence that decays more slowly than any negative power of $n$ : for all $\varepsilon>0$, there exists $c>0$ such that

$$
w_{n}>c n^{-\varepsilon} \text {. }
$$

Therefore, every space $\mathcal{H}_{\mathbf{w}}$ consists of functions analytic in $\Omega_{1 / 2}$, and contains functions that are not analytically extendable to any larger domain. Nonetheless there is a dense subspace of functions in $\mathcal{H}_{\mathbf{w}}$ whose norms can be obtained by 
evaluating suitable integrals over the larger half-plane $\Omega_{0}$. For the case of $\mathcal{H}_{0}$, the following theorem is due to F. Carlson [8]. Note that we do not assume that either side of (1.6) is finite.

Theorem 1.5. Let $f(s)=\sum_{n=n_{0}}^{\infty} a_{n} n^{-s}$ be a function in $\mathcal{D}$ that has $\sigma_{b}=0$. Let $\mu$ satisfy (1.1) and (1.2), and let $w_{n}$ be given by (1.3). Then

$$
\sum_{n=n_{0}}^{\infty}\left|a_{n}\right|^{2} w_{n}=\lim _{c \rightarrow 0^{+}} \lim _{T \rightarrow \infty} \frac{1}{2 T} \int_{-T}^{T} d t \int_{0}^{\infty} d \mu(\sigma)|f(s+c)|^{2} .
$$

Proof. Fix $0<c<1$, and let $\varepsilon>0$. Let $\delta$ be given by

$$
\delta=\frac{\varepsilon}{\left(1+\mu\left[c, \frac{1}{c}+c\right]\right)\left(1+\|f\|_{\Omega_{c}}\right)} .
$$

By Bohr's theorem, the Dirichlet series of $f$ converges uniformly in $\overline{\Omega_{c}}$, so there exists $N$ such that

$$
\left|\sum_{n=n_{0}}^{N^{\prime}} a_{n} n^{-s}-f(s)\right|<\delta \quad \forall s \in \overline{\Omega_{c}}, \forall N^{\prime} \geq N .
$$

Then

$$
\begin{aligned}
\lim _{T \rightarrow \infty} & \frac{1}{2 T} \int_{-T}^{T} d t \int_{0}^{1 / c} d \mu(\sigma)|f(s+c)|^{2} \\
& =\lim _{T \rightarrow \infty} \frac{1}{2 T} \int_{-T}^{T} d t \int_{0}^{1 / c} d \mu(\sigma)\left|\sum_{n=n_{0}}^{N^{\prime}} a_{n} n^{-(s+c)}\right|^{2}+O(\varepsilon) .
\end{aligned}
$$

As

$$
\lim _{T \rightarrow \infty} \frac{1}{2 T} \int_{-T}^{T} d t n^{-(\sigma+i t)} m^{-(\sigma-i t)}=\delta_{m n} n^{-2 \sigma},
$$

we get from (1.7) that

$$
\begin{aligned}
\lim _{T \rightarrow \infty} & \frac{1}{2 T} \int_{-T}^{T} d t \int_{0}^{1 / c} d \mu(\sigma)|f(s+c)|^{2} \\
& =\sum_{n=n_{0}}^{N^{\prime}}\left|a_{n}\right|^{2} \int_{0}^{1 / c} d \mu(\sigma) n^{-2 \sigma-2 c}+O(\varepsilon)
\end{aligned}
$$

for all $N^{\prime} \geq N$. Taking the limit in (1.8) as $c$ decreases to 0 , and noting that $\varepsilon$ can be made arbitrarily small for $N$ large enough, we get

$$
\sum_{n=n_{0}}^{\infty}\left|a_{n}\right|^{2} w_{n}=\lim _{c \rightarrow 0^{+}} \lim _{T \rightarrow \infty} \frac{1}{2 T} \int_{-T}^{T} d t \int_{0}^{1 / c} d \mu(\sigma)|f(s+c)|^{2} .
$$

But this is the same limit as (1.6).

Note that the integrals

$$
\frac{1}{2 T} \int_{-T}^{T} d t|f(\sigma+i t)|^{2}
$$

are monotonically decreasing as a function of $\sigma$, so if $\mu(\{0\})=0$, the monotone convergence theorem yields the following corollary. 
Corollary 1.9. Assume the hypotheses of Theorem 1.5, and also that $\mu(\{0\})=0$. Then

$$
\sum_{n=n_{0}}^{\infty}\left|a_{n}\right|^{2} w_{n}=\lim _{T \rightarrow \infty} \frac{1}{2 T} \int_{-T}^{T} d t \int_{0}^{\infty} d \mu(\sigma)|f(s)|^{2} .
$$

By a multiplier of $\mathcal{H}_{\mathrm{w}}$ we mean a function $\phi$ with the property that $\phi f$ is in $\mathcal{H}_{\mathrm{w}}$ for every $f$ in $\mathcal{H}_{\mathrm{w}}$. It follows from the closed graph theorem that for any multiplier $\phi$, the operator of multiplication by $\phi$, which we denote by $M_{\phi}$, is bounded. It is somewhat surprising that, although the spaces $\mathcal{H}_{\mathrm{w}}$ consist of functions analytic in $\Omega_{1 / 2}$, the multipliers are somehow forced to extend to be analytic on all of $\Omega_{0}$. For the case $\mu=\mu_{0}$, the following theorem is due to Hedenmalm, Lindqvist and Seip [9]. The main idea of our proof is constructing an increasing sequence of semi-invariant subspaces for multiplication operators (1.15).

Theorem 1.11. Let $\mu$ satisfy (1.1) and (1.2), and let $w_{n}$ be given by (1.3). Then the multiplier algebra of $\mathcal{H}_{\mathrm{w}}$ is isometrically isomorphic to $H^{\infty}\left(\Omega_{0}\right) \cap \mathcal{D}$, where the norm on $H^{\infty}\left(\Omega_{0}\right) \cap \mathcal{D}$ is the supremum of the absolute value on $\Omega_{0}$.

Proof. It is clear that any multiplier $\phi$ must be in $\mathcal{D}$, just by considering $\phi(s) \cdot n_{0}{ }^{-s}$. We shall prove the theorem in two parts:

(A) Show that if $\phi \in \mathcal{D}$ has $\sigma_{b}=0$, then

$$
\left\|M_{\phi}\right\|=\|\phi\|_{\Omega_{0}} .
$$

(B) Show that if $\phi$ is a multiplier of $\mathcal{H}_{\mathbf{w}}$, then $\phi$ is analytic and bounded in $\Omega_{0}$.

Proof of (A): Suppose $\phi(s)=\sum_{n=1}^{\infty} b_{n} n^{-s}$ is bounded in all half-planes strictly smaller than $\Omega_{0}$. Let $f(s)=\sum_{n=n_{0}}^{N} a_{n} n^{-s}$ be a finite sum in $\mathcal{H}_{\mathbf{w}}$. Then $\phi f$ has $\sigma_{b}=0$, so by Theorem 1.5

$$
\begin{aligned}
\left\|M_{\phi} f\right\|^{2} & =\lim _{c \rightarrow 0^{+}} \lim _{T \rightarrow \infty} \frac{1}{2 T} \int_{-T}^{T} d t \int_{0}^{\infty} d \mu(\sigma)|\phi(\sigma+c) f(\sigma+c)|^{2} \\
& \leq\|\phi\|_{\Omega_{0}}^{2}\|f\|^{2} .
\end{aligned}
$$

So if $\|\phi\|_{\Omega_{0}}$ is finite, then $M_{\phi}$ is bounded on the dense subspace of $\mathcal{H}_{\mathrm{w}}$ consisting of finite sums, and therefore extends by continuity to be a multiplier of the whole space.

We must show that $\left\|M_{\phi}\right\|$ equals $\|\phi\|_{\Omega_{0}}$. So let us assume that $\left\|M_{\phi}\right\|=1$ and $\|\phi\|_{\Omega_{0}}>1$, and derive a contradiction. (We are not assuming that $\|\phi\|_{\Omega_{0}}$ is necessarily finite.)

For each $\sigma>0$, let

$$
N_{\sigma}=\sup _{t}|\phi(\sigma+i t)| .
$$

By the Phragmén-Lindelöf theorem, $N_{\sigma}$ is a strictly decreasing function of $\sigma$. Indeed, for $\sigma$ very large, $N_{\sigma}$ tends to $\left|b_{1}\right|$ which is less than or equal to $\left\|M_{\phi}\right\|<\|\phi\|_{\Omega_{0}}$, so the conclusion follows by applying the Phragmén-Lindelöf theorem to the function $e^{\varepsilon s} \phi(s)$ for an appropriate choice of $\varepsilon$ on a vertical strip.

Moreover, in each half-plane $\Omega_{c}$ for $c>0$, the Dirichlet series of $\phi$ converges uniformly to $\phi$ by Bohr's theorem, so by Theorem 0.4 , $\phi$ is uniformly almost periodic in $\Omega_{c}$. Therefore there exists $\varepsilon_{1}, \varepsilon_{2}, \varepsilon_{3}, \varepsilon_{4}>0$ so that, for large enough $T$,

$$
\left|\left\{t:|\phi(\sigma+i t)|>1+\varepsilon_{1},-T \leq t \leq T\right\}\right| \geq \varepsilon_{2}(2 T) \quad \forall \varepsilon_{3} \leq \sigma \leq \varepsilon_{3}+\varepsilon_{4} .
$$


(Just find some open set where $|\phi|$ is bigger than one, and by uniform almost periodicty, this will recur as one moves up in the imaginary direction.) Since multiplication by $\phi$ is a contraction, so is multiplication by $\phi^{j}$ for any positive integer $j$. Therefore

$$
\left\|\phi^{j}(s) n_{0}^{-s}\right\|^{2} \leq w_{n_{0}} \quad \forall j \in \mathbb{N} .
$$

So by Theorem 1.5, we conclude that

$$
\begin{aligned}
w_{n_{0}} & \geq \lim _{T \rightarrow \infty} \frac{1}{2 T} \int_{-T}^{T} d t \int_{0}^{\varepsilon_{4}} d \mu(\sigma)\left|\phi^{j}\left(\sigma+\varepsilon_{3}\right)\right|^{2} n_{0}^{-2\left(\sigma+\varepsilon_{3}\right)} \\
& \geq \varepsilon_{2}\left(1+\varepsilon_{1}\right)^{2 j} n_{0}^{-4 \varepsilon_{3}} \mu\left(\left[0, \varepsilon_{4}\right]\right) .
\end{aligned}
$$

As the right-hand side of (1.14) tends to infinity with $j$, we get a contradiction.

Proof of (B): Let $p_{j}$ denote the $j^{\text {th }}$ prime, and let $\mathbb{N}_{N}$ denote the set of positive integers all of whose prime factors are in the set $\left\{p_{1}, \ldots, p_{N}\right\}$ :

$$
\mathbb{N}_{N}=\left\{p_{1}^{\nu_{1}} \cdots p_{N}^{\nu_{N}}: \nu_{1}, \ldots, \nu_{N} \in \mathbb{N}\right\} .
$$

For every positive integer $N$, let $Q_{N}$ denote orthogonal projection from $\mathcal{H}_{\mathrm{w}}$ onto the closed linear span of the functions

$$
\left\{n^{-s}: n \in \mathbb{N}_{N}, n \geq n_{0}\right\} .
$$

Suppose $\phi$ is a multiplier of $\mathcal{H}_{\mathrm{w}}$. Then we have

$$
Q_{N} M_{\phi} Q_{N}=M_{Q_{N}(\phi)} Q_{N}=Q_{N} M_{\phi} .
$$

Moreover, by a truncated version of the Euler product formula, we have that if $f(s)=\sum a_{n} n^{-s}$ is any function in $\mathcal{H}_{\mathrm{w}}$, then

$$
\begin{aligned}
\left|Q_{N}(f)(s)\right| & =\left|\sum_{n \in \mathbb{N}_{N}} a_{n} n^{-s}\right| \\
& \leq\left(\sup \left|a_{n}\right|\right) \prod_{j=1}^{N}\left(1-p_{j}^{-\sigma}\right)^{-1} .
\end{aligned}
$$

So if the coefficients of $f$ are bounded, then $Q_{N}(f)$ is a bounded function in $\Omega_{c}$ for every $c>0$. As $\left(Q_{N} f\right)(s+\varepsilon)=Q_{N}(f(s+\varepsilon))$, if the coefficients of $f$ are $O\left(n^{\varepsilon}\right)$ for every $\varepsilon>0$, we have that $\sigma_{b}\left(Q_{N} f\right) \leq \varepsilon$ for every $\varepsilon>0$, i.e. $\sigma_{b}\left(Q_{N} f\right) \leq 0$. By (1.4), as the weights decay more slowly than any negative power of $n$, it follows that for every $f$ in $\mathcal{H}_{\mathrm{w}}$, the coefficients of $f$ are indeed $o\left(n^{\varepsilon}\right)$ for every $\varepsilon>0$, and a fortiori this hypothesis holds for every $\phi$ in the multiplier algebra of $\mathcal{H}_{\mathrm{w}}$ (since $\phi(s) n_{0}^{-s}$ is in $\left.\mathcal{H}_{\mathrm{w}}\right)$.

Therefore we can conclude that

$$
\sigma_{b}\left(Q_{N} \phi\right) \leq 0 \quad \forall N \geq 1 .
$$

Moreover, by (1.15), multiplication by $Q_{N} \phi$ on $Q_{N} \mathcal{H}_{\mathrm{w}}$ is a compression of $M_{\phi}$, so

$$
\left\|M_{Q_{N} \phi}\right\|_{Q_{N} \mathcal{H}_{\mathrm{w}}} \leq\left\|M_{\phi}\right\|_{\mathcal{H}_{\mathrm{w}}}<\infty .
$$

By repeating the argument in part $(\mathrm{A})$ and estimating

$$
\left\|\left(Q_{N} \phi\right)^{j} 2^{-\nu s}\right\|
$$

for $2^{\nu} \geq n_{0}$, we therefore conclude that

$$
\left\|Q_{N} \phi\right\|_{\Omega_{0}} \leq\left\|M_{\phi}\right\|_{\mathcal{H}_{\mathrm{w}}} \quad \forall N .
$$


By a normal families argument, some subsequence of $Q_{N} \phi$ converges uniformly on compact subsets of $\Omega_{0}$ to some $H^{\infty}\left(\Omega_{0}\right)$ function, $\psi$ say. On compact subsets of $\Omega_{1}$, where the Dirichlet series for $\phi$ converges absolutely, $Q_{N} \phi$ converges uniformly to $\phi$. Therefore $\phi=\psi$, and so $\phi$ must be bounded and analytic in all of $\Omega_{0}$.

It is a theorem of Khintchine and Kolmogorov that if the series $\sum\left|c_{n}\right|^{2}$ is finite, then almost every series $\sum \pm c_{n}$ converges (see e.g. [10] for a proof). It follows that if $\sum a_{n} n^{-s}$ is in $\mathcal{H}_{\mathbf{w}}$, then for almost every choice of signs, $\sum \pm a_{n} n^{-s}$ will converge in $\Omega_{0}$ (and in $\operatorname{cl}\left(\Omega_{0}\right)$ for $\mathcal{H}_{\alpha}$ with $\alpha \geq 0$ ).

\section{Dirichlet-Like SPACES}

Throughout this section, let $\mu$ be a measure satisfying conditions (1.1) and (1.2) of Section 1, and let $w_{n}$ be defined by (1.3) for $n \geq 2$. Define another weight sequence $\mathbf{w}^{\mathrm{b}}$ by

$$
w_{n}^{b}=(\log n)^{2} w_{n}
$$

The space $\mathcal{H}_{\mathbf{w}^{b}}$ is exactly the set of functions whose derivatives are in $\mathcal{H}_{\mathbf{w}}$, and is analogous to the Dirichlet space. We shall prove that the multipliers of $\mathcal{H}_{\mathbf{w}^{b}}$ are contained in the multipliers of $\mathcal{H}_{\mathbf{w}}$. One can prove a similar result for higher order derivatives, but for simplicity we stick to the case of a single derivative.

Theorem 2.1. With notation as above, the multipliers of $\mathcal{H}_{\mathbf{w}^{b}}$ are contractively contained in the multipliers of $\mathcal{H}_{\mathbf{w}}$.

Proof. We shall boot-strap from the following claim.

Claim: There is a constant $K<\infty$ such that, if $\phi$ is a multiplier of $\mathcal{H}_{\mathbf{w}^{b}}$ of norm one, and both $\phi$ and $\phi^{\prime}$ have $\sigma_{b} \leq 0$, then $\phi$ is a multiplier of $\mathcal{H}_{\mathbf{w}}$ of norm at most $K$.

Suppose the claim were proved. Let $\psi$ be any multiplier of $\mathcal{H}_{\mathbf{w}^{b}}$ of norm one. Then for every $N, Q_{N} \psi$ satisfies the hypotheses of the claim, so is a multiplier of $\mathcal{H}_{\mathrm{w}}$ of norm at most $K$. By taking the weak-star limit of a subsequence of $Q_{N} \psi$, we can conclude that $\psi$ is a multiplier of $\mathcal{H}_{\mathbf{w}}$ of norm at most $K$.

To show $K$ must be 1 , assume it were greater. Then there would be a multiplier $\phi$ of $\mathcal{H}_{\mathbf{w}^{b}}$ of norm one, which has norm greater than $\sqrt{K}$ as a multiplier of $\mathcal{H}_{\mathbf{w}}$. By Theorem 1.11,

$$
\left\|M_{\phi^{2}}\right\|_{\mathcal{H}_{\mathrm{w}}}=\left\|M_{\phi}\right\|_{\mathcal{H}_{\mathrm{w}}}^{2}
$$

Then $\phi^{2}$ would be a multiplier of norm one of $\mathcal{H}_{\mathbf{w}^{\mathrm{b}}}$, and have norm greater than $K$ as a multiplier of $\mathcal{H}_{\mathbf{w}}$, a contradiction.

Proof of the Claim. We shall prove the claim with $K=\sqrt{2}$. Suppose the claim is false. Then there is some finite Dirichlet series

$$
f(s)=\sum_{n=n_{0}}^{N} a_{n} n^{s}
$$

in $\mathcal{H}_{\mathrm{w}}$ of norm 1 such that $\|\phi f\|>K$. Let

$$
g(s)=\sum_{n=n_{0}}^{N} a_{n} \frac{1}{\log n} n^{s}
$$

be the primitive of $f$, which is of norm one in $\mathcal{H}_{\mathbf{w}^{b}}$. Let

$$
B=\left\{s \in \Omega_{0}:|\phi(s)|>1\right\} \text {. }
$$


By Theorem 1.5, there exists $c>0$ such that

$$
\lim _{T \rightarrow \infty} \frac{1}{2 T} \int_{-T}^{T} d t \int_{0}^{\infty} d \mu(\sigma)|\phi(s+c) f(s+c)|^{2} \chi_{B}(s+c)>K^{2}-1 .
$$

As $\phi^{j}$ is a multiplier of $\mathcal{H}_{\mathbf{w}^{\text {b }}}$ of norm at most one for every positive integer $j$, we have

$$
1 \geq\left\|\phi^{j} g\right\|_{\mathcal{H}_{\mathbf{w}^{b}}}=\left\|\phi^{j} f+j \phi^{j-1} \phi^{\prime} g\right\|_{\mathcal{H}_{\mathbf{w}}}
$$

Therefore

$$
\begin{gathered}
1 \geq \lim _{T \rightarrow \infty} \frac{1}{2 T} \int_{-T}^{T} d t \int_{0}^{\infty} d \mu(\sigma)|\phi(s+c)|^{2(j-1)} \mid \phi(s+c) f(s+c) \\
+\left.j \phi^{\prime}(s+c) g(s+c)\right|^{2} \chi_{B}(s+c) \\
\geq \lim _{T \rightarrow \infty} \frac{1}{2 T} \int_{-T}^{T} d t \int_{0}^{\infty} d \mu(\sigma) \mid \phi(s+c) f(s+c) \\
+\left.j \phi^{\prime}(s+c) g(s+c)\right|^{2} \chi_{B}(s+c) .
\end{gathered}
$$

By subtracting $\phi f+\phi^{\prime} g$ from $\phi f+j \phi^{\prime} g$ and using Minkowski's inequality on (2.3), we get

$$
4 \geq(j-1)^{2} \lim _{T \rightarrow \infty} \frac{1}{2 T} \int_{-T}^{T} d t \int_{0}^{\infty} d \mu(\sigma)\left|\phi^{\prime}(s+c) g(s+c)\right|^{2} \chi_{B}(s+c)
$$

for all $j$, and so the limit is zero. Therefore by Cauchy-Schwarz, 2.3 becomes

$$
1 \geq \lim _{T \rightarrow \infty} \frac{1}{2 T} \int_{-T}^{T} d t \int_{0}^{\infty} d \mu(\sigma)|\phi(s+c) f(s+c)|^{2} \chi_{B}(s+c) .
$$

This contradicts $(\underline{2.2})$ if $K \geq \sqrt{2}$.

Let us say a Radon measure $\nu$ supported in $\operatorname{cl}\left(\Omega_{0}\right)$ is an $\alpha$-Carleson measure if there exists some constant $C$ such that

$$
\lim _{T \rightarrow \infty} \frac{1}{2 T} \int_{|\Im s| \leq T}|f(s)|^{2} d \nu(s) \leq C\|f\|_{\mathcal{H}_{\alpha}}^{2}
$$

for every finite Dirichlet series $f(s)=\sum_{n=2}^{N} a_{n} n^{-s}$. Then we have

Corollary 2.4. For $0<\alpha<2$, the function $\phi$ is a multiplier of $\mathcal{H}_{\alpha}$ if and only if

(i) $\phi$ is in $\mathcal{D} \cap H^{\infty}\left(\Omega_{0}\right)$

and

(ii) The measure $\left|\phi^{\prime}(s)\right|^{2} d \mu_{\alpha-2}(\sigma) d t$ is $\alpha$-Carleson.

Proof. The necessity of condition (i) follows from Theorem 2.1. For condition (ii), observe that by Cauchy's theorem, if $\phi$ is in $\mathcal{D} \cap H^{\infty}\left(\Omega_{0}\right)$, then $\sigma_{b}\left(\phi^{\prime}\right) \leq 0$.

The function $\phi$ is a multiplier if and only if

$$
\left\|\phi f^{\prime}+\phi^{\prime} f\right\|_{\mathcal{H}_{\alpha-2}} \leq C\|f\|_{\mathcal{H}_{\alpha}}
$$

for every finite Dirichlet series $f$. If $\phi$ satisfies condition (i), then

$$
\left\|\phi f^{\prime}\right\|_{\mathcal{H}_{\alpha-2}} \leq\|\phi\|_{\Omega_{0}}\|f\|_{\mathcal{H}_{\alpha}} \text {. }
$$


So such a $\phi$ is a multiplier if and only if

$$
\left\|\phi^{\prime} f\right\|_{\mathcal{H}_{\alpha-2}}^{2}=\lim _{T \rightarrow \infty} \frac{1}{2 T} \int_{-T}^{T} \int_{0}^{\infty}\left|\phi^{\prime}(s)\right|^{2}|f(s)|^{2} d \mu_{\alpha-2}(\sigma) d t
$$

is controlled by $\|f\|_{\mathcal{H}_{\alpha}}^{2}$, i.e. if and only if condition (ii) holds.

Of course, Corollary 2.4 does not answer the question of what the multipliers of $\mathcal{H}_{\alpha}$ are. For this one needs a characterization of those functions $\phi$ in $\mathcal{D}$ that satisfy condition (ii), as D. Stegenga did for $\mathcal{K}_{\alpha}$ in [15]. We do not know what this condition should be.

\section{A Space of Dirichlet Series with the Pick Property}

Let $\mathcal{H}$ be a Hilbert function space on a set $X$ with reproducing kernel $k$. We say $\mathcal{H}$ has the Pick property if, given any distinct points $\lambda_{1}, \ldots, \lambda_{N}$ in $X$ and any complex numbers $z_{1}, \ldots, z_{n}$, then a necessary and sufficient condition for the existence of a function $\phi$ in the closed unit ball of the multiplier algebra of $\mathcal{H}$ that has the value $z_{i}$ at each $\lambda_{i}$ is that the matrix

$$
\left[k\left(\lambda_{i}, \lambda_{j}\right)\left(1-z_{i} \bar{z}_{j}\right)\right]_{i, j=1}^{N}
$$

be positive semi-definite. We say $\mathcal{H}$ has the complete Pick property if, for any positive integer $s$, any distinct points $\lambda_{1}, \ldots, \lambda_{N}$ in $X$ and any $s$-by- $s$ matrices $Z_{1}, \ldots, Z_{n}$, then a necessary and sufficient condition for the existence of a function $\phi$ in the closed unit ball of the multiplier algebra of $\mathcal{H} \otimes \mathbb{C}^{s}$ that has the value $Z_{i}$ at each $\lambda_{i}$ is that the $N s$-by- $N s$ matrix

$$
\left[k\left(\lambda_{i}, \lambda_{j}\right)\left(I-Z_{i} Z_{j}^{*}\right)\right]_{i, j=1}^{N}
$$

be positive semi-definite. See 2 for a treatment of complete Pick kernels.

For every integer $n \geq 2$, let $F(n)$ be the number of ways $n$ can be factored into a product of integers greater than 1 , where the order matters (so e.g. $F(6)=3$, because of the three factorings $6,2 \times 3,3 \times 2$ ). Let $F(1)=1$. Then the following identity holds [17, 1.2.15]:

$$
\sum_{n=1}^{\infty} \frac{F(n)}{n^{s}}=\frac{1}{2-\zeta(s)} .
$$

For the rest of this section, we shall fix

$$
w_{n}=\frac{1}{F(n)},
$$

and consider the space $\mathcal{H}_{\mathrm{w}}$ (with $n_{0}=1$ ). The kernel function for $\mathcal{H}_{\mathrm{w}}$ is then

$$
k(s, u)=\frac{1}{2-\zeta(s+\bar{u})} .
$$

As the reciprocal of $k$ has only one positive square, it follows from the $\mathrm{M}^{\mathrm{C}} \mathrm{Cullough}-$ Quiggin theorem (see [11, 14], 11), that $k$ is a complete Pick kernel.

Let $\rho=0.86 \ldots$ be the unique real number in $(1 / 2, \infty)$ satisfying $\zeta(2 \rho)=2$. Then the space $\mathcal{H}_{\mathrm{w}}$ is analytic in the half-plane $\Omega_{\rho}$, and in no larger domain because the kernel function blows up on the boundary of $\Omega_{\rho}$.

As $k$ is a complete Pick kernel, there is a realization formula for the multipliers of $\mathcal{H}_{\mathrm{w}}$. 
Theorem 3.4. Let $w_{n}$ be defined by (3.2). Then $\phi$ is in the unit ball of the multiplier algebra of $\mathcal{H}_{\mathrm{w}}$ if and only if there is an auxiliary Hilbert space $\mathcal{L}$, and a unitary $U: \mathbb{C} \oplus \mathcal{L} \rightarrow \mathbb{C} \oplus \ell^{2} \otimes \mathcal{L}$ such that, writing $U$ as

$$
U=\stackrel{\mathbb{C}}{\ell^{2} \otimes \mathcal{L}}\left(\begin{array}{ll}
A & B \\
C & D
\end{array}\right),
$$

we have

$$
\phi(s)=A+B\left(I-E_{s} D\right)^{-1} E_{s} C .
$$

Here $\ell^{2}$ is the space of square summable sequences $\left(c_{n}\right)_{n=2}^{\infty}$, and $E_{s}: \ell^{2} \otimes \mathcal{L} \rightarrow \mathcal{L}$ is the contractive linear operator defined for $s \in \Omega_{\rho}$ on elementary tensors by

$$
E_{s}:\left(c_{n}\right)_{n=2}^{\infty} \otimes \xi \mapsto\left(\sum_{n=2}^{\infty} c_{n} n^{-s}\right) \xi .
$$

Theorem 3.4 is a special case of the realization formula for general complete Pick kernels (see [4, 3], 18, 2]), and we omit the proof.

Notice that $\phi(s)$ defined by (3.5) is guaranteed to be holomorphic only if $\left\|E_{s}\right\|=$ $\sqrt{\zeta(2 \sigma)-1}$ is less than 1, i.e. if $s$ is in $\Omega_{\rho}$. It turns out that $\Omega_{\rho}$ is indeed the maximal domain of analyticity of the multiplier algebra (which we shall denote $\left.\operatorname{Mult}\left(\mathcal{H}_{\mathrm{w}}\right)\right)$.

We shall prove this assertion via interpolating sequences. Let $\left\{\lambda_{i}\right\}_{i=1}^{\infty}$ be a sequence in $\Omega_{\rho}$. We say $\left\{\lambda_{i}\right\}_{i=1}^{\infty}$ is an interpolating sequence for $\mathcal{H}_{\mathbf{w}}$ if the linear operator

$$
\Lambda: f \mapsto\left(\frac{f\left(\lambda_{i}\right)}{\left\|k_{\lambda_{i}}\right\|}\right)_{i=1}^{\infty}
$$

maps $\mathcal{H}_{\mathrm{w}}$ to $l^{2}$ surjectively. We say $\left\{\lambda_{i}\right\}_{i=1}^{\infty}$ is an interpolating sequence for $\operatorname{Mult}\left(\mathcal{H}_{\mathrm{w}}\right)$ if, for any bounded sequence $\left(w_{i}\right)_{i=1}^{\infty}$, there is a function $\phi$ in $\operatorname{Mult}\left(\mathcal{H}_{\mathrm{w}}\right)$ with $\phi\left(\lambda_{i}\right)=w_{i}$.

The sequence $\left\{\lambda_{i}\right\}_{i=1}^{\infty}$ is an interpolating sequence for $\mathcal{H}_{\mathbf{w}}$ if and only if the associated normalized Gram matrix

$$
G_{i j}=\frac{\left\langle k_{\lambda_{j}}, k_{\lambda_{i}}\right\rangle}{\left\|k_{\lambda_{j}}\right\|\left\|k_{\lambda_{i}}\right\|}
$$

is bounded above and below (for a proof of this assertion, see e.g. [13] or [2]). Therefore, every sequence $\left\{\lambda_{i}\right\}_{i=1}^{\infty}$ that tends to the line $\{\sigma=\rho\}$ has a subsequence that is an interpolating sequence for $\mathcal{H}_{\mathbf{w}}$ : just choose the $n^{\text {th }}$ element in the subsequence so that the first $n-1$ entries in the $n^{\text {th }}$ row and $n^{\text {th }}$ column of $G$ are smaller in modulus than $2^{-n}$, and then the off-diagonal entries will have Hilbert-Schmidt norm smaller than 1 .

It is a theorem of D. Marshall and C. Sundberg 12 that, for any space with the Pick property, the interpolating sequences for the Hilbert space and for its multiplier algebra coincide (the proof can also be found in [2]). Coupling this with the previous observation, we conclude that for every boundary point of $\Omega_{\rho}$, there is a sequence that converges to that boundary point and that is interpolating for $\operatorname{Mult}\left(\mathcal{H}_{\mathbf{w}}\right)$. Choosing $w_{i}$ to alternate 1 and -1 , we conclude that there is a function in $\operatorname{Mult}\left(\mathcal{H}_{\mathrm{w}}\right)$ that cannot be continued analytically to any neighborhood of that boundary point. Indeed we can do more. Provided $\Re\left(\lambda_{i}\right)$ converges to $\rho$ quickly enough, the sequence $\left\{\lambda_{i}\right\}$ will be interpolating for $\mathcal{H}_{\mathrm{w}}$ regardless of how 
$\Im\left(\lambda_{i}\right)$ is chosen. So we can choose a sequence that accumulates on the whole line $\{\Re(s)=\rho\}$, and conclude that there is a single function $\phi$ in $\operatorname{Mult}\left(\mathcal{H}_{\mathbf{w}}\right)$ that has $\Omega_{\rho}$ as its maximal domain of analyticity. Thus we have proved:

Theorem 3.6. With notation as above, the largest common domain of analyticity of the multipliers of $\mathcal{H}_{\mathrm{w}}$ is $\Omega_{\rho}$.

Is there a better way to describe the interpolating sequences for $\operatorname{Mult}\left(\mathcal{H}_{\mathrm{w}}\right.$ ) (or $\mathcal{H}_{\mathrm{w}}$ ) than requiring that the normalized Grammian be bounded above and below?

Conjecture 3.7. Let $w_{n}$ be given by (3.2). Then the sequence $\left\{\lambda_{i}\right\}_{i=1}^{\infty}$ is interpolating for Mult $\left(\mathcal{H}_{\mathbf{w}}\right)$ if and only if

(i) there exists some constant $C$ such that, for any $i \neq j$, there is a function $\phi$ in $\operatorname{Mult}\left(\mathcal{H}_{\mathrm{w}}\right)$ of norm less than $C$ with $\phi\left(\lambda_{i}\right)=0$ and $\phi\left(\lambda_{j}\right)=1$

and

(ii) there exists some constant $C$ such that, for any $f$ in $\mathcal{H}_{\mathrm{w}}$,

$$
\sum_{i=1}^{\infty} \frac{\left|f\left(\lambda_{i}\right)\right|^{2}}{\left\|k_{\lambda_{i}}\right\|^{2}} \leq C\|f\|_{\mathcal{H}_{\mathbf{w}}}^{2}
$$

Condition (i) is clearly necessary, and condition (ii) is equivalent to requiring that the normalized Grammian be bounded. So the question is whether weak separation (condition (i)) and the Carleson measure condition (ii) suffice. The conjecture is a special case of Question 9.57 in [2].

\section{REFERENCES}

[1] J. Agler and J.E. McCarthy. Complete Nevanlinna-Pick kernels. J. Funct. Anal., 175(1):111124, 2000. MR 2001h:47019

[2] J. Agler and J.E. McCarthy. Pick Interpolation and Hilbert Function Spaces. American Mathematical Society, Providence, 2002. MR 2003b:47001

[3] C-G. Ambrozie and D. Timotin. On an intertwining lifting theorem for certain reproducing kernel Hilbert spaces. Integral Equations 83 Operator Theory 42(4):373-384, 2002. MR 2002m:47012

[4] J.A. Ball, T.T. Trent, and V. Vinnikov. Interpolation and commutant lifting for multipliers on reproducing kernel Hilbert spaces. In Operator Theory and Analysis, pages 89-138. Birkhäuser, Basel, 2001. MR 2002f: 47028

[5] F. Bayart. Opérateurs de composition sur des espaces de séries de Dirichlet et problèmes d'hypercyclicité simultanée. Ph.D. thesis, Université des Sciences et Technologie de Lille, 2002.

[6] A.S. Besicovitch. Almost periodic functions. Cambridge University Press, London, 1932

[7] H. Bohr. Über die Bedeutung der Potenzreihen unendlich vieler Variabeln in der Theorie der Dirichletschen Reihen $\Sigma a_{n} / n^{s}$. Nachr. Akad. Wiss. Göttingen math.-Phys. Kl., pages 441-448, 1913.

[8] F. Carlson. Contributions á la théorie des séries de Dirichlet, Note I. Ark. Mat., 16(18):1-19, 1922.

[9] H. Hedenmalm, P. Lindqvist, and K. Seip. A Hilbert space of Dirichlet series and systems of dilated functions in $L^{2}(0,1)$. Duke Math. J., 86:1-37, 1997. MR 99i:42033

[10] P. Lévy. Sur les séries dont les termes sont des variables éventuelles indépendentes. Studia Math., 3:119-155, 1931.

[11] S.A. McCullough. Carathéodory interpolation kernels. Integral Equations and Operator Theory, 15(1):43-71, 1992. MR 92m:47030

[12] D. Marshall and C. Sundberg. Interpolating sequences for the multipliers of the Dirichlet space. Preprint; see http://www.math.washington.edu/ marshall/preprints/preprints.html, 1994.

[13] N. K. Nikol'skiı. Treatise on the Shift Operator: Spectral Function Theory, volume 273 of Grundlehren der mathematischen Wissenschaften. Springer-Verlag, Berlin, 1985. 
[14] P. Quiggin. For which reproducing kernel Hilbert spaces is Pick's theorem true? Integral Equations and Operator Theory, 16(2):244-266, 1993. MR 94a:47026

[15] D. Stegenga. Multipliers of the Dirichlet space. Illinois Math. J., 24:113-139, 1980. MR 81a:30027

[16] E.C. Titchmarsh. The theory of functions. Oxford University Press, London, 1932.

[17] E.C. Titchmarsh. The theory of the Riemann Zeta-function, Second edition. Oxford University Press, Oxford, 1986. MR 88c:11049

[18] A.T. Tomerlin. Products of Nevanlinna-Pick kernels and colligations. Integral Equations and Operator Theory, 38:350-356, 2000. MR 2001j:47009

Department of Mathematics, Washington University, St. Louis, Missouri 63130

E-mail address: mccarthy@wustl.edu 